\title{
LOS DOMINIOS DEL PENSAMIENTO CRÍTICO: UNA LECTURA DESDE LA TEORÍA DE LA EDUCACIÓN
}

\author{
The domains of the critical thinking: a reading from \\ the Theory of the Education
}

\section{Les domaines de la pensée critique: une lecture depuis la Théorie de l'Éducation}

\author{
Antonio Víctor Martín García* y Óscar Barrientos Bradasic** \\ * Universidad de Salamanca. Facultad de Educación. Departamento de Teoría \\ e Historia de la Educación. Paseo de Canalejas, 169. 37008 Salamanca. \\ Correo-e: avmg@usal.es \\ ** Universidad de Magallanes. Correo-e: obradasic@botmail.com
}

Fecha de recepción: marzo de 2009

Fecha de aceptación definitiva: julio de 2009

Biblid [(1130-3743) 21, 2, 2009, 19-44]

RESUMEN

En el artículo se realiza un análisis de cómo el pensamiento crítico es un factor presente en diversas e importantes corrientes filosóficas y psicoeducativas. En el trabajo se identifican tres grandes marcos descriptivos del pensamiento crítico: el de la lógica argumentativa, el marco axiológico y la perspectiva cognitiva. Desde la teoría educativa se analizan todos estos aportes y la compleja madeja de conceptos y líneas teóricas en la que se inscribe este constructo y, a partir de ahí, se plantea orientar la acción pedagógica hacia la formación del pensador crítico. Se concluye identificando rasgos del pensador crítico en tanto que actor que construye y registra las potencialidades de la cognición y las enlaza con la praxis, esto es, con una acción reflexiva orientada a la toma de decisiones. 
Palabras clave: pensamiento crítico, pensador crítico, lectura crítica, lógica argumentativa, sistemas alternos de pensamiento, habilidades de pensamiento, disposiciones de pensamiento crítico.

\section{SUMMARY}

In this article an analysis is made of how critical thinking is a factor in different and important trends in philosophy and educational psychology. Here we identify three large descriptive frameworks for critical thinking: that of argumentative logic, the axiological framework and the cognitive perspective. All these contributions, as well as the complex bundle of theoretical lines and concepts in which this construct is inscribed, are analyzed from the point of view of theory of education. Based on this, we then discuss how to orient pedagogical action towards training in critical thinking. We conclude by identifying the traits of a critical thinker as an actor who constructs and registers the potentialities of cognition and relates them to praxis, that is, with a reflexive action oriented towards decision-making.

Key words: critical thought, critical thinker, critical reading, argumentative logic, alternative systems of thought, thinking skills, critical thinking dispositions and habilities.

\section{SOMMAIRE}

Dans cet article on analyse comment la pensée critique constitue un facteur présent en divers et importants courants philosophiques et phsyco-éducatifs. Trois grands cadres descriptifs y sont identifiés: celui de la logique argumentative, le cadre axiologique et la perspective cognitive. Tous ces apports ainsi que l'ensemble complexe de concepts et de lignes théoriques où est inscrite cette hypothèse sont analysés à partir de la théorie éducative, de sorte à orienter l'action pédagogique vers la formation du penseur critique. En guise de conclusion, les traits du penseur critique -en qualité d'acteur qui construit et enregistre les potentialités de la cognition et les associe à la praxis avec une action réflexive orientée vers la prise de décisions- sont identifiés.

Mots clés: la pensée critique, un penseur critique, lecture critique, logique argumentative, des systèmes alternatifs de pensée, habileté de pensée, des dispositions de pensée critique.

\section{INTRODUCCIÓN}

Existe la creencia de que el pensamiento crítico, en su creciente abstracción, tiene aplicaciones recientes en el marco del contexto educativo. En parte eso es así porque se tiene conciencia clara y acotada del término solamente desde un 
contexto contemporáneo. No obstante, el pensamiento crítico ha sido siempre un paradigma presente en el campo educacional (quizá bajo otros nombres o sugerido por otros soportes teóricos). De hecho, el conjunto de nociones y categorías que gravitan alrededor del concepto de pensamiento crítico reviste una red muy compleja de saberes, a los cuales sólo se puede acceder, de una forma coherente, por medio de un enfoque interdisciplinario que conecte paradigmas que vienen tanto de la epistemología como del abordaje psicológico. Un análisis, siquiera somero, de ambas líneas nos permitirá valorar el interés pedagógico de un concepto clave para la teoría y la práctica educativa en cualquiera de los niveles educativos.

El pensamiento crítico se ha tornado una tradición de investigación y de enseñanza de origen filosófico y de desarrollo multidisciplinario. Tal es así, que el pensamiento crítico se ha configurado como un constructo muy fecundo, tanto en el campo de la educación, del desarrollo social e incluso de la organización empresarial. Podría decirse que el pensamiento crítico ha sido objeto de estudio desde ámbitos disciplinares teóricos que, a su vez, se constituyen en campos de observación: la filosofía (que ha concentrado sus energías en el aspecto ontológico y epistemológico), la psicología (que ha investigado las capacidades y funciones implicadas en esta estrategia cognitiva y, fundamentalmente, con los mecanismos de resolución de problemas) y los profesionales de la educación (que han proyectado estas reflexiones en el marco de la escolarización y la ampliación de las prácticas pedagógicas transformadoras).

Al margen de la multiplicidad de visiones en torno al pensamiento crítico, podemos acotar algunos aspectos como parte de esta reflexión, en realidad, más vinculados a los acuerdos que a posicionamientos diametralmente divergentes.

En primer término, admitir que el pensamiento crítico es una operación de naturaleza cognitiva y, por lo tanto, se halla estrechamente emparentada con la gama más amplia de accesos al conocimiento. Esto también indica que no es la única ni la exclusiva facultad de esta índole, queremos decir que pensamiento crítico no es pensamiento mágico, ni pensamiento lógico, ni razonamiento geométricoespacial, etc. Eso sí, en los márgenes de abstracción colinda con otras formas y estilos cognitivos.

El segundo punto a considerar es que pensar críticamente la realidad implica necesariamente acceder a soluciones epistemológicas por sistemas alternos de pensamiento, es decir, por caminos de enriquecimiento cognitivo destinados a repensar las estructuras de toda racionalidad mecánica. En otras palabras, existe la necesidad de poner en duda las propias percepciones, evitando el escepticismo rotundo o la criticidad compulsiva, y profundizando la disensión y la independencia cognitiva.

En tercer lugar, otro de los rasgos recurrentes del pensamiento crítico es su carácter argumentativo, ya que su operación más elocuente sería la de fundamentar, encontrando en las líneas de acción que ofrece la figura del argumento, las bases de una comprensión capaz de derribar determinados paradigmas y de erigir otros como nuevos puntos de apoyo o pilares de comprensión.

En cuarto, y tomando un concepto de Freire, el pensamiento crítico tendría como uno de sus rasgos básicos la capacidad de coexistencia. Esto implica una 
noción amplia de los saberes y una aceptación de la objetividad inclusiva y no como el factor que define la verdad. Es decir, hablaríamos de un eclecticismo participativo, más centrado en la búsqueda de fundamentos dinámicos que en dogmas o axiomas, en el marco de una idea dialéctica de la verdad.

Por último, otro aspecto fundamental sería su orientación diagnóstica-propositiva. El pensamiento crítico no sólo abordaría las representaciones de la realidad a través del análisis y el contraste, sino que su ejercicio implicaría un lenguaje proposicional que en gran medida funcionaría como sustituto de la experiencia con material cualitativo.

Pues bien, nos proponemos en este trabajo considerar todos estos sentidos, agrupados aquí en tres grandes dominios, con la mirada puesta en sus derivaciones e implicaciones para la teoría y la práctica educativa.

\section{El DOMINIO DE LA RAZÓN}

El análisis realizado en este apartado nos conduce a tres sublíneas claras que conectan pensamiento crítico y educación desde la perspectiva del conocimiento filosófico. Líneas difícilmente separables que, únicamente desde un mero afán expositivo, distinguimos en los siguientes apartados. Nos referimos, por un lado, a la línea de la argumentación y la lógica formal, la vía de conexión del pensamiento crítico con el lenguaje y los aspectos textuales, y, en tercer lugar, la perspectiva que sitúa al concepto en la interacción sujeto-mundo.

\subsection{Pensamiento crítico y educación argumentativa (razón y lógica formal)}

Como hemos indicado, la sola definición de pensamiento crítico ha ido acumulando un amplio espectro de significados culturales, provenientes de diversas tradiciones filosóficas que, en muchos casos, tienen su asidero en el tronco de las disciplinas retóricas y en la figura argumentativa. Se entiende que, en el llamado pasado clásico, gran parte del bagaje retórico no se reducía al mero embellecimiento de la palabra, no existiendo separación entre la forma y el contenido. La retórica estaba más bien vinculada a la oratoria y en especial al ámbito tribunicio. El divorcio ocurre en la Edad Media, donde se subordina gran parte de estas categorías a los fenómenos de causalidad aristotélicos. Precisamente, Aristóteles distinguía entre episteme y doxa, catalogando el primero como un conocimiento argumentado y sólido, en contraste a un conocimiento inconsistente, basado en apreciaciones sin soporte racional ni experimental. Este primer acercamiento -muy rescatado posteriormente en la cosmovisión medieval- no afirma de ningún modo que lo objetivo sea necesaria y exclusivamente lo verdadero. No obstante, esta noción admite el carácter irreductible de la cognición como catalizador de las relaciones entre los objetos aprendidos por la realidad y el sujeto que las conceptualiza. 
Cuando Descartes, separa -por lo menos en términos de razonamiento- la conciencia de la existencia y sostiene la supremacía de aquélla como fenómeno donde se resume, en gran parte, la cabalidad de las cosas, deriva el problema a que el carácter crítico del pensamiento no radica en el estímulo externo de los objetos que nos circundan, sino en la propia conciencia. Sin embargo, el conocimiento racional es inseparable del sustrato de los arrebatos emocionales, de los sentimientos, de las pasiones; éstos no pueden considerarse meras transformaciones que en lo posible habría que eliminar a fin de obtener un conocimiento objetivo, sino que integran la base del conocimiento mismo como premisas inevitables. La corporalidad define y decide la actividad cognoscitiva y la propia actividad mental.

El pensamiento crítico es sugerido más claramente por los postulados de Kant, en su constante tensión entre sujeto/objeto. El problema kantiano gravita en torno a la máxima de que "la Ilustración es la salida del hombre de su autoculpable minoría de edad". Por ello, en el aspecto crítico del proceder humano apela al sentido de la razón para superar esa minoría, pero también advierte los peligros de una racionalidad emancipadora, ya que la Ilustración antes de ser un fenómeno vinculado a la epistemología sería un atributo de la libertad.

El pensamiento crítico (desde la mirada kantiana) es una actividad en la que colaboran tanto lo empírico como lo racional y donde resulta fundamental distinguir con claridad lo que corresponde a cada uno. Lo que la ciencia pone en evidencia es el carácter activo de la conciencia. La ciencia es expresión de la acción de la conciencia. Al reconocerse el carácter activo de la mente, se descubre la forma a través de la cual la conciencia y los objetos contribuyen en la tarea del conocimiento. La conciencia contribuye con las relaciones, la experiencia con los objetos relacionados y representa el foco desde el cual la experiencia se organiza, se estructura, alcanza unidad, síntesis. La conciencia ordena la experiencia sería probablemente la base kantiana del pensamiento crítico.

Desde esta idea, la propuesta kantiana es el racionalismo crítico, entendiendo por ello el uso de la razón fundado previamente en un análisis de los poderes y límites de la razón. En gran medida, los aportes de Kant representan a la vez un importante esfuerzo por superar y conciliar tanto el dualismo filosófico como las dos corrientes de pensamiento a que él daba lugar.

Al hacerlo, sin embargo, transfiere al interior de su concepción los propios términos de la oposición que pretende superar. Kant reemplaza el dualismo cartesiano de la sustancia en un dualismo de tipo experiencial: la experiencia del conocimiento, de la teoría, ligada a la razón pura, y la experiencia del comportamiento humano, de la moral y de la fe, ligada a la razón práctica. Teoría y práctica, conocimiento y acción, serán los términos de un dualismo corregido, pero aún no superado. La conciencia, dice Kant, es ciertamente el primer acto de la razón y en ella se funda últimamente toda experiencia, pero el segundo acto es la intuición y 
el tercero, el conocimiento ${ }^{1}$. Como podemos observar, la noción de pensamiento crítico sigue estrechamente signada a los límites cognitivos.

A esa concepción corresponden, en Alemania, la teoría diltheyana de la comprensión cuya circularidad - de la que no se puede escapar- recusa cualquier intento de construir el conocimiento de manera progresiva y unidimensional. Si el hombre, como enseña Dilthey (1944) comprende en cuanto vive, de antemano se invalida la empresa de construir sin supuestos el conocimiento.

Considerada en términos pedagógicos, la línea argumentativa del pensamiento crítico propone un tipo de educación basada en competencias argumentativas, propositivas e interpretativas. De este modo, trata de promover un tipo de práctica educativa en la que los alumnos aprendan a saber justificar, apoyar, desarrollar una idea, saber valorar y considerar diversas alternativas, convencer de algo a alguien y, de manera particular también, saber contraargumentar.

En la práctica se trataría de que los alumnos aprendan las reglas y los diferentes tipos de argumentos causales: analogías; generalizaciones, argumentos de naturaleza esencial, argumentos categóricos, argumentos no proposicionales, tales como las apelaciones emotivas, etc.; la elaboración de diagramas de argumentos, conexiones causales y de argumentos analógicos. Por otro lado, el desarrollo del pensamiento creativo desde este primer dominio que consideramos que conllevaría una praxis educativa centrada en el desarrollo de la lógica formal (silogismos, uso y evaluación de inferencias inductivas, etc.) además del reconocimiento de las falacias, el uso y el control de la ambigüedad, definiciones y la construcción de argumentos ${ }^{2}$.

\subsection{Pensamiento crítico y educación para la lectura crítica del texto}

Algunos autores se han aproximado a la noción de pensamiento crítico desde la perspectiva específica del lenguaje. M. Heidegger, por ejemplo, en El ser y el tiempo (1927) plantea que el mundo no es un fenómeno situado fuera de la conciencia para ser racionalmente analizado, en el marco de una lectura contemplativa del sujeto, dicho de otro modo no es algo de lo cual podamos salir para asimilarlo. Es más,

1. A partir del influjo kantiano, y a lo largo de los siglos XIX y principios del XX, diversas corrientes filosóficas plantearon algunos abordajes a la noción de pensamiento crítico. Un estudio exhaustivo obligaría a considerar aportaciones tomadas de la tradición dialéctica desde la óptica idealista de Hegel (1770-1831) a la vertiente materialista de Karl Marx (1818-1883); la corriente analítica de Bertrand Russell (1872-1970) y la tradición fenomenológica-hermenéutica de Husserl (1859-1938); Dilthey (1833-1911); la Escuela de Baden (1900-1914) con sus principales líderes: Windelband (1848-1915) y Rickert (1863-1936). Por esta misma época asistimos a las contribuciones de Feuerbach (1804-1872) con su empirismo positivista primitivo; Comte (1798-1857) positivismo; Stuart Mill (1806-1873); Durkheim (1858-1917); Weber (1864-1920); el positivismo lógico del Círculo de Viena con el liderazgo de Schlick (1892-1936), relacionado con el Círculo también está Mach (1838-1916); Gödel (1906); Popper (1902), etc.

2. Vid. Herrick (1991); Racofal (1990); Rudinow y Barry (1994); Grennan (1997). 
para Heidegger el sujeto emerge de una realidad que nunca puede objetivar del todo e incluye al objeto en su simbiosis. De esta forma denominará "precomprensión" para entender el proceso por el cual, antes de pensar sistemáticamente, llevaríamos en nuestro interior un espectro de suposiciones no dichas en los vínculos prácticos que nos ligan al mundo. Por ello, el pensamiento crítico sería el diálogo que funde estas concepciones en un todo comprensivo porque donde hay lenguaje hay mundo. Utiliza, específicamente, la expresión "abrirse al ser" para determinar lo que bien podría llamarse pensamiento crítico o dimensión crítica del lenguaje.

Scriven y Fischer (1997) han insistido en que el pensamiento crítico es una competencia académica estrechamente emparentada con la escritura y con la lectura. Cuando hablamos de lectura crítica inevitablemente agregamos la palabra texto, en su sentido más amplio, es decir, como un planteamiento estructural cuyas partes son congruentes y están interrelacionadas. No olvidemos que la etimología de la palabra "texto" proviene de la voz latina texere que quiere decir tejido. Los vínculos entre discurso y pensamiento crítico orientan el problema al espacio dinámico que transcurre entre lo conceptual y el lenguaje, llevando a entender la noción de lectura crítica como el soporte cognitivo por antonomasia. Es más, algunos como Adam Schaf (1973) han llegado a afirmar que no existe pensamiento sin el formato discursivo o por lo menos lingüístico.

La distinción entre lectura crítica y pensamiento crítico la realiza Daniel J. Kurland (1995) a partir de la necesidad perentoria por establecer categorías conceptuales que no sólo funcionen en el plano de las ideas sino también en su consiguiente proyección en determinadas líneas de acción. En este caso, el proceso de lectura de la realidad. En primer lugar, la distinción de Kurland se restringe a los límites que conectan el plano semántico o del significado con el ámbito específico de la textualidad. En otras palabras, enlaza la idea de comprensión con la búsqueda de claves certeras para acceder a una sistematización o conceptualización.

Lo que convierte en útil la distinción, se encuentra en recordar que debemos leer cada texto por sus propios méritos, sin que se interpongan el conocimiento previo del lector o los puntos de vista que sobre él se puedan tener. Al tiempo que se deben evaluar las ideas mientras se lee. Si bien se trata de una separación necesaria para limitar la idea de pensamiento crítico y para que ésta no se vuelva demasiado expansiva, es restringida al estricto ámbito de la coherencia y no admite aspectos de tanta relevancia como los contextos, las narrativas, la pragmática del discurso, la comunicación dialógica, todos ellos, fundamentales en la configuración de los denominados sistemas alternos de pensamiento ${ }^{3}$.

3. "Cuando adoptamos el punto de vista monístico, rechazamos la afirmación de que el lenguaje y el pensamiento pueden existir separadamente y con independencia entre sí. Desde luego, nos referimos a un pensamiento específicamente humano, en otras palabras, un pensamiento conceptual. Así afirmamos que en el proceso de cognición y comunicación, pensar y utilizar un lenguaje son elementos inseparables de un todo. La integración es tan perfecta y la interdependencia tan precisa que 
Es sabido que esta idea de pensamiento crítico únicamente vinculante a la generación del lenguaje es rebatida por Chomsky ${ }^{4}$, quien sostiene que la mediación discursiva no agota todas las posibilidades del conocimiento, constituyéndose el discurso en una posibilidad (no la única) y enlazando la actividad cognitiva a horizontes más amplios, incluso inefables. De la misma forma sostiene que pensar y experimentar son aspectos que no se pueden divorciar con tanta soltura. La idea de Chomsky de que el pensamiento crítico genera formas discursivas solamente desde el estatuto de las llamadas estructuras profundas ha sido muchas veces consignada como una profundización del clásico circuito comunicativo. En lo fundamental, se trata de describir los dispositivos críticos que gravitan en torno a la idea de texto, mensaje, pensamiento y discurso como un viaje semántico, que va clasificando los estímulos de la realidad e ingresándolos a un campo de conceptualización.

Lo que se denomina pensamiento crítico involucraría también a un conjunto de percepciones de la realidad. De hecho, las formas de experiencia que facilitan los sentidos harían más complejos los límites del propio significado. Los procesos psicológicos son formas de representación y como tales, incluyen todo lo que puede abarcar el mundo empírico; este punto de apoyo nos lleva a sostener que el pensamiento crítico al convertirse en discurso no sólo opera una transformación, sino también una reducción. La experiencia siempre requiere un contenido.

En torno a esta relación complementaria entre pensamiento crítico y lectura crítica, Freire (1997) agrega un concepto que unifica ambas ideas y al que denomina exigencia ontológica, ya que el aspecto crítico sería el umbral para intercambiar contenidos con su naturaleza histórica y con sus condiciones objetivas. Freire lo ejemplifica a través de la separación ideológica entre texto y contexto, pero también sitúa al pensamiento crítico como el dispositivo que contrasta un supuesto fatalismo. Más claramente, lo que sugiere Freire es que el pensamiento crítico es ante todo un constructor y generador de discursos transformadores. La potencialidad discursiva del pensamiento crítico no sólo tiene que ver con un diagnóstico de la realidad, sino también como atributo esencial de una conciencia en camino a su propia liberación. En ese aspecto, el concepto adquiere ribetes fenomenológicos.

ninguno de los elementos puede darse jamás independientemente, en una forma "pura". Precisamente por este motivo las funciones del pensamiento y el lenguaje no pueden tratarse por separado, y no digamos contrastadas entre sín. Vid. Schaff $(1997,118)$

4. Curiosamente Chomsky expresa su discrepancia en el prólogo a la obra de ScHAF (1971): "¿Es cierto, por ejemplo que los seres humanos pensamos necesariamente con un lenguaje? Se nos ocurren de inmediato ejemplos evidentes a favor de lo contrario. Nuestra única evidencia de cualquier sustancia es instrospectiva, y sin duda la introspección dice que cuando pienso en un viaje a París o una excursión a las Montañas Rocosas, los pocos fragmentos de monólogo interior que pueden detectarse apenas transmiten, o incluso sugieren, el contenido de mi pensamiento. Al esforzarse por resolver un problema matemático, uno es con frecuencia consciente del papel de una intuición física, geométrica, que es apenas expresable en palabras, ni siquiera con esfuerzo y atención”. Veáse también: CHOмsкY (1983, 1988). 
Esa línea teórica de conexión del pensamiento crítico y los aspectos textuales fue recogida también por Hans Georg Gadamer (1975) en su libro Verdad y método, donde examina el enfoque crítico de la realidad desde su dimensión textual e interpretativa. Por ello, el pensamiento crítico genera un discurso comprensivo de lo que él denomina la verdad profunda del texto "que sirve de puente entre la distancia temporal que separa al intérprete y al texto, superando así la enajenación del significado sufrida por el texto". La visión hermenéutica de Gadamer, en el fondo, observa que el pensamiento crítico es generador de discurso porque su idea de la historia es la de un diálogo viviente entre presente, pasado y futuro. La labor de la interpretación crítica del texto sería remover los escombros que arroja el significado en su largo proceso comunicativo.

Para Gadamer, la raíz del pensamiento crítico (y en eso coincide con Habermas y Freire) radica en la figura del diálogo, capaz de imbricar las expectativas discursivas a una mirada más global y sistemática, que estaría, por así decirlo, empotrada en las estructuras de comunicación de las sociedades. Su punto de apoyo, a pesar de aceptar la ruptura interpretativa, es la reverencia que hace a la tradición que en su parecer "encuentra su justificación fuera de los argumentos de la razón". Es decir, Gadamer anula casi en su totalidad la función de aquello que Derrida definirá como "precomprensiones" y sostiene que el prejuicio puede ser un puente de acceso a la interpretación de los discursos que nos conecta a la ansiada tradición, que, como vemos, es un concepto más abstracto de lo que parece.

También desde el campo de la gramática transfrástica (o lingüística textual) de Teum Van Dijk \& Kinstch (1983) la producción textual es un proceso estratégico donde operan mecanismos simultáneos y flexibles en la construcción del mensaje. Pensamiento crítico textual viene a ser entonces la representación en paralelo del contenido de los mensajes, sus formas léxicas (palabras), sus formatos sintácticos (orden en la oración), la ideología (estructuración de diferentes visiones de mundo) y formatos fonológicos (representación de sonidos).

La dimensión crítica del lenguaje, en tanto construcción de discurso, es el objeto de análisis en la obra de Ludwig Wittgenstein. En el Tractatus plantea que «el mundo es la totalidad de los hechos, no de las cosas". Pensamiento crítico y discurso encuentran en la obra de Wittgenstein una simbiosis perfecta, al considerar que el lenguaje constituye un mapa del mundo, por ello sus límites vienen a ser también

5. Ludwig Wittgenstein (Viena, Austria, 26 de abril de 1889; Cambridge, Reino Unido, 29 de abril de 1951) fue un filósofo fundacional con respecto a la teoría del lenguaje y tuvo una importancia radical en los campos de la filosofía, la lógica y la música. Realizó estudios de ingeniería, participó en la Primera Guerra Mundial y trabajó como maestro, jardinero y profesor en la Universidad de Cambridge. Sus tres obras más importantes son el Tractatus Logico-Philosophicus que influyó mucho a los positivistas lógicos del Círculo de Viena, Las Investigaciones Filosóficas y Los cuadernos azul y marrón, ambas póstumas. Fue discípulo de Bertrand Russell en el Trinity College de Cambridge. 
los límites del mundo. Debido a esto, los problemas filosóficos serían más bien perplejidades, callejones sin salida sugeridos de la razón.

Desde esta visión, se sostiene que el significado de las palabras y su contenido semántico conllevan implícitamente su función y contextualidad. El lenguaje, en su dimensión crítica, es la totalidad de las proposiciones significativas que en su globalidad dinámica nombra los contextos de uso. Su mirada crítica es elocuente porque en virtud de la relación isomórfica entre lenguaje y mundo la verdad se constituye como la relación recíproca y complementaria entre el sentido de lo representado y una proposición o un hecho.

En efecto, en su Tratado Lógico-Filosófico, publicado en 1921, Wittgenstein sostenía que había un grupo numeroso de palabras que designaban directamente partes sustanciales de la realidad. Este supuesto hecho era la base del positivismo lógico y, por derivación, de las definiciones operacionales, ya que enlazaba las proposiciones con la propia realidad. Pero desde 1930 en adelante, Wittgenstein comienza a cuestionar, en sus clases en la Universidad de Cambridge, sus propias ideas, y a sostener, poco a poco, una posición que llega a ser radicalmente opuesta: niega que haya tal relación directa entre una palabra y un objeto; llegando a afirmar que las palabras no manifiestan referentes directos; sostiene que los significados de las palabras se encuentran determinados por los diferentes contextos en que ellas son usadas; que los significados no tienen límites rígidos, y que éstos están formados por el contorno y las circunstancias en que se emplean las palabras; que, consiguientemente, un nombre no puede representar o estar en lugar de una cosa y otro en lugar de otra, ya que el referente particular de un nombre se halla determinado por el modo en que el término es usado. En resumen, Wittgenstein dice que en el lenguaje jugamos con palabras y que usamos a éstas de acuerdo a las reglas convencionales preestablecidas en cada lenguaje. Todas estas ideas aparecerán luego en su obra póstuma Investigaciones Filosóficas (1953).

En estos pasos de conciencia crítica ubica los llamados juegos de lenguaje, que vendrían a ser los entramados en los cuales las palabras encuentran su semanticidad más profunda y sus implicaciones más estructurales y, por lo tanto, determinantes. Por ejemplo, el juego de lenguaje de la ética, de la ciencia, de la estética, de la religión, serían parte de la construcción de una crítica cultural.

Desde el interés pedagógico podemos concluir que la generación de discurso conlleva elementos contextuales y de significación donde el pensamiento crítico funciona como eje pragmático. De este modo, el problema de la generación de discursos en el pensamiento crítico se enlaza en muchas oportunidades a la búsqueda de una supuesta esencia del lenguaje, que, por ejemplo, Wittgenstein denomina infructuosa, trocando esa tarea por la búsqueda de la descripción de las reglas que configuran los contextos y significados del lenguaje.

Para algunos autores reconocidos en este campo, como Van Dijk (1980), es necesario distinguir determinadas estrategias que deberían ser desarrolladas en la formación de pensadores críticos: estrategias de producción interactivas y pragmáticas, que 
incluyen acciones, planificaciones de la producción y planes discursivos; estrategias de producción semántica, que incluyen estrategias para la construcción de macroestructuras semánticas (es decir, temas o significados globales de los mensajes); estrategias para la construcción de microestructuras semánticas (significados locales) y estrategias para establecer la coherencia específica de un pasaje.

Estas estrategias de la lingüística textual abarcan el espectro más amplio de operaciones textuales y, pese a su enfoque clasificador, tienden a seguir extendiendo los puentes entre texto y pensamiento crítico. La misión del pensador crítico no sería la de descubrir nada que se encuentre oculto, sino presentar con claridad la textualidad significativa con la cual interactuamos.

El proceso formativo derivaría aquí en el entrenamiento del alumno en tratar de despejar esa suerte de "embrujamiento" conceptual sistematizado por las llamadas proposiciones atómicas. Por ejemplo, Wittgenstein identifica las siguientes proposiciones atómicas:

- La representación de hechos atómicos: aquellos que forman parte del lenguaje significativo. Dichas proposiciones, en tanto que se refieren a hechos, son susceptibles de ser verdaderas o falsas.

- La no representación de hechos atómicos: aquellos que no pertenecen al lenguaje significativo con sentido, es decir, que carecen totalmente de significación. Éstas a su vez se subdividen en tautologías y contradicciones (de manera que estas proposiciones serán siempre verdaderas las unas y siempre falsas las otras, aunque de un modo bastante distinto que las anteriores, puesto que «no representan ningún posible estado de cosas") y absurdas e insensatas (estas proposiciones no pueden ser ni verdaderas ni falsas, sino absurdas. Éstas no son proposiciones en sentido estricto, sino que se trata de seudoproposiciones o scheinsätze). Para Wittgenstein (2000) los problemas filosóficos surgen cuando se intenta expresar lo que no se puede decir, olvidando los requisitos que dotan de significado a las proposiciones.

En suma, este análisis ofrece una dimensión crítica del lenguaje que aborda tanto los límites de la cognición como sus propios mecanismos de desarticulación. De esta manera, el significado de una palabra o de una proposición estaría determinado por su uso específico en el lenguaje.

\subsection{Pensamiento crítico y educación para la lectura crítica del contexto}

El aspecto central de esta línea de trabajo podríamos resumirlo en la idea de que el pensamiento crítico no sólo formula un lenguaje sino también un discurso, es decir, un espacio de negociaciones simbólicas con el tiempo y con los contextos en los que nos situamos. Por ello, se entiende fundamental la capacidad interrogativa del pensador crítico para cuestionar los procesos históricos y descubrir sus complejos mecanismos ocultos. 
El enfoque de Habermas (1984, 1990) amplía tanto los conceptos de pensamiento crítico como de discurso. Para Habermas, en el pensamiento crítico coexiste una racionalidad cognitiva instrumental (centrada en las formas de representación y sus evidentes posibilidades de intervención), la moral práctica (que establecería los acuerdos y desacuerdos de aspectos como el bien común y las prácticas consuetudinarias) y la estética-expresiva (que se concentraría en las manifestaciones culturales y su correspondiente carga simbólica).

En su análisis el proyecto de modernidad cultural formulado por los filósofos del Iluminismo en el siglo XVII se basaba únicamente en el desarrollo de una ciencia objetiva, una moral universal, una ley y un arte autónomos y regulados por lógicas propias, liberando el potencial cognitivo de todo principio metafísico. Es decir, el Iluminismo albergaba la ingenua esperanza de que la ciencia y el arte fuesen los encargados de comprender la totalidad del mundo y la felicidad de los individuos. Si bien Habermas (1989a, 1989b, 2000) no observa ese proceso emancipador de la ilustración como una derrota, sino como algo que se encuentra inconcluso, habría que precisar los elementos de continuidad planteados por Habermas. El pensamiento crítico es una operación creadora que interviene en planos diferentes y sucesivos de conocimiento y significación. Por esta razón no se encuentra ajena a los escenarios o contextos de producción en que se plantea. La prepotencia del método sobre el objeto deriva de esta consideración reificadora (o quizá redentora) de la realidad social. La vía crítica no es sólo formal; no sólo se limita a la reflexión sobre los enunciados, sino que también debe ser crítica con respecto al objeto del que dependen todos estos momentos. Es decir, del sujeto y los sujetos vinculados a la ciencia organizada.

Habermas critica al positivismo su estrecha concepción de la observación científica, al reduccionismo que implica sólo al análisis del comportamiento observable, a las variables aislables de experimentación y a sujetos intercambiables a voluntad. Frente a ello propone una teoría dialéctica, la cual acepta realidades incontrolables y hallazgos no corroborables empíricamente. Así entonces, dicha teoría empatiza con la Hermenéutica, ya que procede en términos del sentido que los propios agentes le atribuyen a la acción social.

Para Habermas, las normas no son ni verdaderas ni falsas, sino posiciones; de ahî que los enunciados normativos no tengan por base el conocimiento sino la decisión.

Desde esta posición, es difícil, pero elocuente, establecer cuál es el margen de pensamiento crítico que se establece en contextos demasiado habituales donde los grupos privilegiados social y económicamente imponen la valía de sus expresiones comunicativas en detrimento de sectores con menos capacidad de acción y diálogo ${ }^{6}$. De alguna manera los dispositivos críticos de Habermas restan importancia a la figura del poder.

6. En esta línea se sitúa, con mayor carga ideológica, la referencia de los universos espirituales a las condiciones materiales y económicas de los hombres que los crean. Cuando Marx (1970) dice "no es 
Las miradas de la posmodernidad también han abordado la idea de pensamiento crítico, llevándolo al ámbito de un diagnóstico de las verdades absolutas. Para Nietzsche, las corrientes de la historia moderna se caracterizaban por ser irónicas y dialécticas, considerando los ideales de integridad del alma y la voluntad de verdad como propios del cristianismo. En su lectura, el pensamiento crítico choca con una tradición conservadora decimonónica: "ser mediocre es la única moral que tiene sentido". Si para Benjamin la actitud crítica de la realidad estaría marcada por un discurso que situado desde el presente involucre una actualización de los paradigmas del pasado, para Nietzsche, el pensamiento crítico es una consecuencia reactiva a lo que denomina "la parodia del pasado" y que se traduce en la aterradora certeza de que ningún papel social de los tiempos modernos podrá ajustarse nunca a la perfección.

¿Dónde radicaría entonces la conciencia del pensamiento crítico en una cosmovisión nihilista que observa el estatuto moral y las normas sociales como construcciones arbitrarias? Justamente ahí, en su fase devastadoramente rotunda de los paradigmas de la cultura cristiana occidental y la construcción de un nuevo horizonte de expectativas que suprima la culpa y la virtud como medidas prescriptivas. Digamos que el aporte de Nietzsche a la idea de pensamiento crítico es tan polémico como revelador. Ofrece una alternativa que implica una revisión extrema totalmente rotunda, que consiste en destruir las viejas catedrales del pasado y erigir en su lugar templos a la propia humanidad, entendida ésta como la certeza absoluta de la libertad.

Esta proposición, por totalizante que sea, la vemos parcialmente en algunas fases de los existencialistas y poco en Lyotard o Foucault, quienes recogen su legado sólo en la fase de profundización crítica, pero niegan la posibilidad de cualquier tipo de libertad. Foucault incluso habla de una "jaula de hierro" como la capacidad de la condición humana por adaptarse a las rejas. Es poco lo que se puede hacer cuando la sociedad y la educación son consecuencia de las estructuras, instituciones creadas por la burguesía para sembrar la reproducción genealógica y el control ideológico. Abiertamente cree que el poder consume todas las posibilidades de expansión, incluso en sus dimensiones menos aparentes, más subterfugias.

No se trata de entender que el ya gastado debate entre modernidad y posmodernidad sea el componente de fondo en una visión actual del pensamiento crítico. Se trata de entender que una mirada crítica del lenguaje se puede caracterizar por una visión apocalíptica de la sociedad como un entramado complejo donde las estructuras de poder lo regulan todo o que, por otro lado, hay quienes ven el pensamiento crítico en su naturaleza dialéctica, como enriquecedor y transformador del contexto.

la conciencia lo que determina la vida, sino la vida lo que determina la conciencia", esto significa que también él niega una conciencia autónoma y, por lo tanto, un conocimiento sin supuestos. 
La tesis básica aquí es que los discursos se encuentran tensionados por el poder, y que el interminable diálogo de la historia ha sido, en más de una ocasión, un monólogo donde se expresa sólo la ideología hegemónica de los poderosos. Es evidente que el margen de pensamiento crítico tiende a mermarse en contextos demasiado habituales donde los grupos privilegiados imponen la valía de sus expresiones comunicativas en detrimento de sectores con menos capacidad de acción y diálogo.

El pensador estructuralista Louis Althusser (1975) desarrolla en su trabajo Ideología y aparatos estatales una lectura del rol del pensamiento crítico muy vinculada a los alcances críticos de Lacan y su visión de la mente humana como un flujo constante de ideas, a veces inconsciente, que determinan la actitud del hombre en la sociedad. Althusser parte del cuestionamiento de que el grueso de los seres humanos se someten a los poderes políticos dominantes. Por ello intenta desentrañar los mecanismos que funcionan para que eso ocurra y concluye que esos individuos son funcionales a una estructura social específica. Por ende, todos los elementos propios de una sociedad determinada, como la escuela, los deberes cívicos, los hábitos de urbanidad son meras reproducciones del sistema social.

En esa visión, la ideología ocuparía todo el conjunto de creencias y prácticas que hacen posible la reproducción del sistema social impuesto por el poder. Esta fase final hace creer a Althusser que el "sujeto" nace cuando reconoce su funcionalidad en el engranaje social, en el contexto ${ }^{7}$.

En resumen, en las tradiciones filosóficas anteriormente mencionadas encontramos que el pensamiento crítico en su aplicación al ámbito educativo se define por las relaciones que establece con los límites del lenguaje, la razón y la relación entre el sujeto y el mundo por un lado, pero, por otro, vemos que se insinúa un perfil más valorativo que mira a una educación transformadora y liberadora, al que nos referimos, más específicamente, en el siguiente apartado.

7. Althusser asigna una importancia radical al pensador crítico, en este caso, los intelectuales, ya que todos los que detentan la generación del conocimiento en función del poder son ideólogos conscientes o inconscientes de dicho esquema global. Por esta última vía llega a dos conclusiones: "Los profesores de filosofía son profesores, es decir intelectuales empleados en un sistema escolar dado, sometidos a ese sistema, ejerciendo en su masa una función social de inculcación de los valores de la ideología dominante. Que pueda existir al margen en las instituciones escolares u otras que permite a ciertos profesores individuales volver a su enseñanza y sus reflexiones contra esos valores establecidos no modifica el efecto de la masa en la función profesoral filosófica. Por eso la filosofía dominante cuyos representantes o portadores, incluso en su libertad crítica, son en su masa profesores de filosofía, está sometida a aquella filosofía dominante, la que Marx definió desde la ideología de la clase dominante, (Althusser [1975, 80]). 


\section{El DOMINIO AXIOLÓGICO}

A mediados del siglo XVIII se proyecta la idea de pensamiento crítico a la escuela. No quiere esto decir en absoluto que esta proyección sea cabal, sino que se inicia un sesgo de visiones tendentes a cuestionar el excesivo racionalismo y un horizonte valórico más amplio dispuesto a reestructurar un cierto perfil axiológico. La instancia específica que distingue a la teoría y al pensamiento crítico es el interés emancipador, en un intento de suprimir la injusticia social. De aquí deriva su no conformismo, su beligerancia en pro de una sociedad buena y racional, y la constante atención a los desarrollos de la realidad.

\subsection{Pensamiento crítico y educación crítica}

Probablemente el primer ataque importante al modelo educativo imperante es el que estipula Rousseau, quien formula la llamada corriente "naturalista" en el Emilio. Su fervorosa creencia en que la educación debe estar cerca del medio natural lo hace acercarse al mentado mito del buen salvaje y a una creciente sensibilidad con los valores humanos y con la pobreza. En el contexto de Rousseau el pensamiento crítico sería el dispositivo necesario para rechazar los cánones autoritarios del sistema educativo imperante. La repercusión de las ideas de Rousseau será fundamental en los grandes reformadores del siglo XIX, en la Escuela Nueva, en la Escuela Progresista de Dewey y en la Institución Libre de Enseñanza.

De hecho, la educación crítica (a partir del legado de Rousseau) será fundamental también en el ímpetu renovador de Pestalozzi quien incorpora la noción de pedagogía social, vista como la necesidad de un sistema educativo al servicio del pueblo. Pensamiento crítico -en el contexto de la llamada educación elemental de Pestalozzi- es el principio de naturalidad en el cual la naturaleza humana se asume como una unidad indivisible.

No obstante, la tesis de Pestalozzi no logra resolver la globalidad del ejercicio dialéctico que envuelve el proceso de enseñanza y el pensamiento crítico -en su lectura- se subordina al desarrollo de las facultades físicas que acompañan el progreso intelectual y a los límites de una moral religiosa. Ese retorno al idealismo romántico será rescatado y potenciado por Froebel quien prácticamente homologará pensamiento crítico como una fase más de una dinámica lúdica del proceso enseñanza-aprendizaje ${ }^{8}$.

Los antecedentes de Rousseau y Pestalozzi, así como también Froebel, Ellen Key y, por supuesto, la escuela de Yasnai Polai inspirada en los preceptos

8. La pedagogía de Froebel, a base de juegos musicales, el dibujo y el uso de materiales específicos en los que se trataba de inculcar en los niños la forma, el color, el movimiento y la materia, significaba, en el fondo, la propuesta de prácticas que ayudarían al niño a cuestionar de una forma más cabal la realidad. 
espirituales del gran escritor ruso León Tolstói, servirán para que el pensamiento crítico tenga verdaderamente su lugar e importancia definida, en el ámbito educativo, a través de la Escuela Nueva. Los planteamientos de la Escuela Nueva incluyen al pensamiento crítico como el mecanismo más eficaz para despejar la espesa hojarasca del enciclopedismo. La lectura crítica se focaliza en el programa didáctico, donde abordajes coexistentes forman los criterios educativos de la escuela.

Así, por ejemplo, para Montessori el pensamiento crítico en el marco de la escuela se vincula a una idea sensorial de las prácticas educativas, orientadas más al descubrimiento personal y la autonomía cognitiva. Desde esta lectura, el pensamiento crítico tiene una orientación paidocéntrica y vitalista, pues implica herramientas de cuestionamiento de la realidad para derivar en una nueva concepción del aprendizaje, creando una fusión urgente entre pensamiento y contexto.

El pensamiento crítico, desde este marco teórico, se erige como un concepto expansivo, en tanto orienta la adquisición cognitiva a espacios de problematización del aprendizaje y no a una lectura vertical de los procesos educativos. No obstante, este abordaje está limitado a una crítica de la institución educativa desde el interior de la propia escuela y no amplía sus campos de acción a una crítica social más global y aguda de los cimientos ideológicos y de las estructuras profundas sobre las cuales se construye.

Esta visión vitalista y activa de la educación adquiere su máxima concreción en la obra de J. Dewey, para algunos considerado el padre de la tradición moderna del pensamiento crítico. De hecho lo planteó como una de las principales metas educativas del siglo XX. El aporte de Dewey (1933) en torno a la proyección del pensamiento crítico en el marco de la escuela está contenido en el libro ¿Cómo pensamos? Allí asocia la labor educativa a la necesidad de suspender juicios, el ejercicio de una mente abierta conjugando el perfil reflexivo con un escepticismo sano, capaz de alentar un cuestionamiento permanente y dialéctico de los espacios que le toca habituar al individuo-aprendente. Dewey sugiere que el pensamiento crítico no sólo posee componentes intelectuales sino también emocionales.

Más aún, Dewey distinguía entre pensamiento reflexivo y pensamiento crítico. El primero sería la función principal de la inteligencia mientras que el segundo estaría abocado al ámbito específico del aprendizaje como procesos de acción y organización de experiencias verdaderas. En los sugestivos planteamientos de Dewey -más allá del excesivo clasicismo de algunos de sus postulados- se conecta la idea de pensamiento crítico con el campo de los valores y de una moral aprendida mediante la observación y la participación en un control social. "Pensamiento crítico es consideración activa, persistente y cuidadosa de una creencia o de una forma supuesta de conocimiento, a la luz de los fundamentos que las apoyan y de las ulteriores conclusiones que se desean extraer" dice textualmente Dewey ${ }^{9}$.

9. Dewey (1933). 
Al plantear que el pensamiento crítico es un proceso activo, Dewey separa esta modalidad cognitiva del proceso pasivo o receptivo que se manifiesta cuando uno recibe las ideas o la información por parte de otras personas. Con esto se da a entender que son importantes las razones que se tienen para creer en algo y las implicaciones de esas creencias.

Como bien podemos observar, uno de los aspectos que dirige y ordena los lineamentos del pensamiento crítico en la práctica educativa se relaciona con una idea de la escuela como un espacio de libertad cognitiva y ante todo, antiautoritario. Precisamente, el movimiento autoritario, representado en su génesis por Robin, los preceptos filosóficos del literato ruso León Tolstói y el español Ferrer Guardia tiene una patente voluntad de autogestión. De este modo el pensamiento crítico comienza a asociarse a la idea de escuela libertaria y a la urgente necesidad antropológica de presentar una visión nueva del hombre.

En esta línea, podría decirse también que algunos de los postulados del imaginario ideológico anarquista, con respecto al pensamiento crítico, coinciden con la llamada educación no directiva de C. Rogers (1949), fuertemente inspirada por la psicología humanista: un sentido crítico y no direccional de los contenidos de la enseñanza orientados al autoaprendizaje.

Pero no es el único punto común en el cual se decanta la práctica del pensamiento crítico en el ámbito de la escuela. Por ejemplo, el modelo historicista de Goodman y la teoría de la desescolarización de Ilich y Reimer manifiestan una importante valoración del pensamiento crítico, centrando los polos enseñanzaaprendizaje en el modelo formativo como corazón de la verdadera institución educativa.

Naturalmente, el pensamiento crítico (como la mayoría de los conceptos derivados de las ciencias sociales) estuvo marcado por la aportación marxista, entendiendo esta destreza cognitiva como un instrumento que enseña a apreciar un diagnóstico de la realidad y a observar las condiciones desiguales de la sociedad capitalista. Por ello se vinculará el pensamiento crítico a la idea de los medios de producción y la educación como elemento fundamental de transformación. Marx y Engels hablan de una educación omnilateral.

Esto tiene sus proyecciones en la escuela de trabajo de Blonskij que pretendía unificar la interpretación radical del trabajo productivo con los lineamientos de la educación intelectual, vieja paradoja del marxismo al intentar conciliar trabajadores manuales e intelectuales. Posteriormente, Bordieu y Passeron desarrollarán el pensamiento crítico con la reproducción de las relaciones entre las clases sociales.

La mirada pedagógica nos señala aquí diferentes vertientes en torno al rol del pensamiento crítico en la escuela. Todas ellas proponen el rol activo de un diagnóstico nuevo capaz de romper las estructuras anquilosadas de la educación tradicional, pero en su dinámica muchas veces plantean elementos diferentes e incluso contrarios. Por ejemplo, es casi imposible divorciar el concepto de pensamiento crítico que plantea la pedagogía anarquista del marco teórico que orienta 
y construye su sentido de la libertad. En este caso, los aportes de Bakunin, Owen, Proudhon y Saint-Simon coinciden en una fuerte crítica a los valores de la tradición burguesa, amparados en el atrapante problema de la culpa cristiana y de la constitución de los medios de producción al servicio de los grandes poderes económicos. Hay en esta mirada un rechazo tajante a la construcción idealista de la conciencia y el retorno a un cientificismo capaz de comprender de forma más fehaciente el núcleo de las contradicciones humanas ${ }^{10}$. Por ello, se propone un sistema educativo en el modelo de la comuna autogestionaria, de carácter mutual, a la manera de los ya conocidos falansterios de Fourier. El pensamiento crítico pasa a ser un método activo y dinámico para la comprensión de las reales funciones de una sociedad justa y una forma de organización más evolucionada.

\subsection{Pensamiento crítico y educación emancipadora}

Otros autores han ampliado algunas de estas cuestiones introduciendo matices de gran interés para la teoría educativa. Por ejemplo, A. Guiddens, P. Freire, E. Glasser, H. Gabennesch, D. Walton, R. Paul, etc., próximos a las corrientes de educación liberadora, de pensamiento crítico reflexivo, de los enfoques constructivistas o de creatividad, entre otros, han contribuido a delimitar también los contornos de esta nueva dirección transformadora del pensamiento crítico. En su sentido más profundo, se trata de una dirección de pensamiento y acción educativa social en la que confluyen múltiples articulaciones, bajo un tronco común de inspiración humanista.

Giddens (1985) habla de pensamiento crítico desde el carácter dual de la acción transformadora, que define como estructura y agencia humana, llevado por el móvil de un diálogo integrador centrado en la búsqueda de la verdad a través del consenso ${ }^{11}$. Para Paulo Freire (1997), la noción de pensamiento crítico es mucho más que una destreza cognitiva, supone el paso fundamental al momento de una comprensión totalizante del mundo y lo enlaza con la configuración de la conciencia y con la dialéctica conciencia/mundo y subjetividad/objetividad. Sitúa este concepto como un espacio de definición tensionado por las perspectivas mecanicistas e idealistas. Los primeros sostendrían que la conciencia sería una especie

10. «Si el pueblo debe preservarse del gobierno de los sabios, con mayor razón debe presumirse contra el de los idealistas inspirados. Cuanto más sinceros son esos creyentes y esos poetas del cielo, más peligrosos se vuelven. La abstracción científica, lo he dicho ya, es una abstracción racional, verdadera en su esencia, necesaria a la vida de la que es la representación teórica, la conciencia. Puede, debe ser absorbida y digerida por la vida. La abstracción idealista, dios, es un veneno corrosivo que destruye y descompone la vida, que la falsea y la mata" (BAKunin, 2003, 63).

11. Giddens (1985). 
de construcción arbitraria del mundo, mientras que los segundos plantean que cambiar la realidad implica previamente "purificar" la conciencia moral.

Reflexionar, evaluar, programar, investigar, transformar son especificidades de los seres humanos en el mundo con el mundo. La vida se torna existencia y el entorno, mundo, cuando la conciencia del mundo, que implica conciencia de mí, al emerger ya se encuentra en relación dialéctica con el mundo. La cuestión de la tensión conciencia-mundo, que implica relaciones mutuas, ya llevó a Sartre a decir que "conciencia y mundo se dan al mismo tiempo". Las relaciones entre ambos son naturalmente dialécticas, sin importar la escuela filosófica de quien las estudia (Freire, 1997, 23).

Para Freire es el pensamiento crítico (sugerido por la conciencia) quien genera la posibilidad de reinventar el mundo, considerando la Historia como posibilidad y no como determinismo. Freire considera su experiencia pedagógica como una transformación cultural de los oprimidos, su mensaje se dirige a la conciencia humana ya que desde ahí es posible la liberación. Distingue tres estados de la conciencia que corresponden a sociedades y culturas diferentes: la conciencia intransitiva de sociedades cerradas; la transitiva de sociedades en las que los dominados viven en inferioridad; y la conciencia crítica de sociedades con un desarrollo económico y democrático caracterizadas por el diálogo y la libertad.

La pedagogía de Freire puede denominarse como de la conciencia, ya que el mundo y ella se constituyen mutuamente y al unísono. Los cambios en las sociedades y los estados de conciencia pueden sobrevenir por motivos sociohistóricos, de la educación o de la propia intervención. En otras palabras, pensamiento crítico y transformación serían conceptos recíprocos e irreductibles.

Glaser (1941), otro de los estudiosos que ha insistido en vincular el pensamiento crítico a los paradigmas de la educación transformadora, contempla el pensamiento crítico como un esfuerzo persistente para examinar cualquier creencia o cualquier forma de conocimiento a la luz de las evidencias que la sustentan. Dicho de otra manera como una actitud de estar dispuesto a considerar de manera reflexiva los problemas y las materias que se relacionan con las experiencias personales.

\subsection{Pensamiento crítico y educación para la búsqueda de la verdad}

En el artículo titulado "Pensamiento crítico ¿para qué sirve?" publicado en The Skeptical Inquirer, el sociólogo Howard Gabennesch (1992) define esta destreza cognitiva así: «El pensamiento crítico consiste en el uso de nuestras aptitudes racionales, ideas y valores para acercarnos a la verdad tanto como sea posible".

En la misma línea, para Kurland (1995) el pensamiento crítico se relaciona tajantemente tanto a la racionalidad como a la honestidad intelectual, en el marco del seguimiento sistemático a las evidencias que arroja la realidad. Esta visión se sostiene sobre la máxima de que la búsqueda de la verdad prima sobre tener 
la razón y el concepto retorno a su base inicial, centrada en la problemática argumentativa ${ }^{12}$.

Para Richard Paul (1992), uno de los principales teóricos sobre el tema, el pensador crítico mejora la cualidad de su pensamiento al tomar conciencia de las estructuras inherentes al acto de pensar, imponiendo norma intelectual sobre ellas. El pensamiento crítico es también entendido por Paul como metacognición o, dicho de otro modo, como la forma por la cual los paradigmas educativos reflexionan en torno a sus propios límites y a sus mecanismos de articulación y desarticulación. Para Paul, el pensamiento multilógico requiere la habilidad de pensar de manera precisa e imparcial en un contexto de puntos de vista y marcos de referencia opuestos o contradictorios que caracterizan la realidad de las relaciones interpersonales en la vida cotidiana, por ello observa que gran parte de los actos de la vida cotidiana se basan en creencias apoyadas en la experiencia, costumbres, convenciones, etc., que no han sido examinadas racionalmente o son producto del encubrimiento de mecanismos individuales y sociales no conscientes. Estas creencias y valores pueden presentar contradicciones o inconsistencias que requieren de clarificación, desmitificación, interpretación y reanálisis.

En la base de la formación de estos valores se entremezclan las disposiciones, creencias, afectos y juicios que dan lugar, según M. Lipman (1992, 306), a dos posiciones básicas: la posición de conservación que puede perfectamente traducirse como la preservación de los valores tradicionales de la sociedad. Esta posición se apoyaría en la idea o postulado de que es necesario transmitir los códigos morales considerados valiosos, de generación en generación, garantizando de esa forma la integridad y la continuidad social. Lo característico de esta posición es el respeto por unos ideales, asociados a la identidad nacional, que son la herencia del pasado y al mismo tiempo una guía fiable para el futuro. Por otro lado habla de la posición de preparación para el cambio centrado en la expresión de valores de diversidad e innovación cultural. Su postulado aquí es que la habilidad para la discusión argumentada pasa a primer plano de atención más que el contenido de los valores.

Estas visiones teóricas, no obstante, coinciden en el carácter transversal y axiológico del pensamiento crítico, que no sólo razona, evalúa y analiza, sino también define experiencialmente y aprecia, todo ello desde un fondo valórico. Dicho de otro modo, observar críticamente la realidad conlleva también pronunciarse en torno a ella.

12. «El pensamiento crítico es una forma de pensar de manera responsable relacionada con la capacidad de emitir buenos juicios. Es una forma de pensar por parte de quien está genuinamente interesado en obtener conocimiento y buscar la verdad y no simplemente salir victorioso cuando está argumentando". Traducción realizada por EDUTEKA del documento escrito por Daniel J. KuRLAND "Critical Reading v/s Critical Thinking". En http://www.critical-eading_thinking.htm. 
Del mismo modo que en los dominios anteriores, también en el ámbito más específico de la psicología el pensamiento crítico ha sido definido y sistematizado por diversos autores y desde distintos ángulos (Dressel y Mayhew [1954], MacPeck [1981], Beber [1985], Nickerson [1987], Wade y Travis [1987], Chafe [1988], Kurfiss [1988], Hudgins [1989], Siegel [1989], Paul [1990], Stratton [1999], Ennis [1996], Faccione [2000], Halpern [2003], etc.).

La posición más extrema entre los expertos sería la de quienes entienden que la noción de pensamiento crítico es una especie de entelequia, ya que el pensamiento por antonomasia es crítico, y no requeriría ese apellido. Sin embargo, lo común es aceptar que en la estructura del pensamiento conviven una amplia gama de contenidos epistemológicos que actúan de manera conjunta e interrelacionada. Estos contenidos son los conceptos (teorías, axiomas, leyes, principios, etc.), las interpretaciones e inferencias, la información (datos, hechos, observaciones..., los marcos de referencia y perspectivas, el análisis y la resolución de problemas, y, por supuesto, los propósitos iniciales y las implicaciones o consecuencias finales del acto de pensar que pueden generar la toma de decisiones). La cognición viene a ser, desde este esquema, el eje por el cual los puentes entre el pensamiento y las representaciones de la realidad encuentran puntos de acuerdo y complementariedad.

A partir de la base del carácter multidimensional y complejo del pensamiento, el aspecto crítico del mismo consideraría algunas destrezas cognitivas específicas relacionadas a su vez con determinados procesos cognitivos. La cuestión se plantea aquí en determinar cuáles son estas habilidades o destrezas cognitivas específicas y propias del pensamiento crítico y sobre todo si es posible entrenarlas o potenciarlas mediante secuencias racionales de acción educativa.

Respecto a la primera cuestión, y considerando tan sólo algunos de los autores más representativos en este tema, Marzano, Brand, Hughes, Jones, Presseinsen, Ranking y Suhor (1988), identifican cinco dimensiones de pensamiento: metacognición (la conciencia sobre lo que se piensa en un momento dado basada en el conocimiento y autocontrol); el pensamiento creativo y crítico; procesos de pensamiento (formación de conceptos, principios de comprensión, resolución de problemas, toma de decisiones, investigación, composición y expresión oral); destrezas fundamentales (orientarse en la tarea, recolectar datos, recordar, organizar, generar, integrar y evaluar); por último, el contenido del conocimiento.

Por su parte, Costa (1989) clasifica las estrategias cognitivas en tres estadios claramente diferenciados: 1 . Datos de ingreso (Habilidades para recopilar y recordar información); 2. Procesamiento de información (Habilidades para dar sentido a la información adquirida; 3. Resultados (Habilidades para aplicar y evaluar acciones en situaciones reales o hipotéticas).

Giancarlo y Facione (2001) identifican seis habilidades centrales para el pensamiento crítico: 1. análisis, 2. inferencia, 3. interpretación, 4. evaluación, 5. explicación y 6. autorregulación. 
"El Informe Delphi»"13 aventura un consenso en torno a la noción de pensamiento crítico, caracterizando sus estadios y sus líneas de acción en dos fases claramente determinadas:

1) Ser el producto de un esfuerzo de interpretación, análisis, evaluación e inferencia de las evidencias. 2) Ser explicado o justificado, por consideraciones evidenciables, conceptuales, contextuales y de criterios, en las que se fundamenta.

En resumen, se asume que este tipo de pensamiento conjuga dos componentes principales: el componente cognitivo (habilidades o destrezas) y el componente actitudinal (disposiciones).

La línea de trabajo en torno a las disposiciones de pensamiento está siguiendo, en los últimos años, un importante recorrido investigador. Se entiende que las actitudes, los valores e inclinaciones de una persona afectan el pensamiento crítico, asumiendo que estas disposiciones de pensamiento tienen el valor transcendental de activar, en situaciones de la vida cotidiana, las destrezas o habilidades de pensamiento.

Para David Perkins, Eileen Jay y Shari Tishman (1993) las disposiciones de pensamiento, en términos generales, son tendencias hacia patrones particulares de comportamiento intelectual. Desde un modelo que denominan "concepción triádica de las disposiciones de pensamiento" (sensibilidad -la percepción de un comportamiento específico-; inclinación -el ímpetu sentido hacia un comportamiento- y habilidad - la capacidad básica de llevar a cabo el comportamiento-) estos autores identifican siete disposiciones: la disposición de ser abierto y aventurero, la disposición de preguntarse, de encontrar problemas e investigar, la disposición de construir explicaciones y comprensiones, la disposición de hacer planes y ser estratégico, la disposición de ser intelectualmente cuidadoso, la disposición de buscar y evaluar razones, la disposición de ser metacognitivo.

Perkins, Jay y Tishman (1993) prefieren expresar estas disposiciones en términos de: disposición a plantear y explorar problemas, disposición a criticar y testar teorías y explicaciones, disposición a buscar múltiples perspectivas y posibilidades y disposición a ser juicioso y reflexivo.

Desde la filosofía de la educación, Robert Ennis (1987, 1990, 1993) identifica hasta catorce disposiciones de pensamiento crítico. En el estudio de Giancarlo y Facione (2001), utilizando el Inventario de disposiciones para el pensamiento crítico (CCTDI) identificaron siete disposiciones hacia el pensamiento crítico:

13. En 1990 participaron en un panel un total de 46 expertos de Estados Unidos y Canadá representando a muchas disciplinas de humanidades, ciencias sociales y educación. La monografía fue publicada en ese mismo año bajo el título: Critical Thinking: A Statement of Expert Consensus for Purposes of Educational Assessement and Instruction. Este proyecto fue construido y patrocinado por la Asociación Norteamericana de Filosofía. Delphi (Asociación Filosófica Americana), Pensamiento Crítico: una Declaración de Consenso de Expertos con fines de evaluación e instrucción educativa. "El Informe Delphi». Comité Preuniversitario de Filosofía (ERIC Doc., n. ${ }^{\circ}$ ED 315 423), 1990. 
1) búsqueda de la verdad, 2) mente abierta, 3) capacidad de análisis, 4) pensamiento crítico sistemático, 5) seguridad en su razonamiento, 6) curiosidad y 7) madurez para emitir juicios.

Al segundo aspecto que señalábamos como nuclear en esta mirada cognitiva sobre el pensamiento crítico, y más allá de la definición u operativización del constructo o de la propia medida del mismo, esto es, el grado en que puede enseñarse a pensar críticamente, nos referiremos más específicamente en el siguiente apartado.

\section{A MOdO DE RECAPITULACIÓN: LA FORMACIÓN DEL PENSADOR CRÍTICO}

Sabemos que la acumulación de conocimiento no constituye, en sí mismo, garantía de desarrollo de un abordaje crítico de la realidad. Dicho de otro modo, la educación formal, en cualquiera de sus niveles, ha tendido, en general, a la adquisición de contenidos propios de las diversas materias y, en menor medida, a la formación de pensadores. A pesar de ello, o quizá precisamente por esta razón, al menos en las dos últimas décadas se ha producido un notable aumento de cursos, materiales curriculares y programas que ayudan al alumno a pensar. Sin embargo, como señala Lipman $(1997,160)$, no es suficiente con esto, la cuestión se plantea en términos de enseñar a pensar críticamente.

Conforme a la definición de Ennis pensar críticamente es decidir en forma razonada y reflexiva qué creer o qué hacer. De manera más precisa, Kurland (1995) entiende que pensar críticamente significa confiar en la razón como principal patrón de conducta y ampliar la cantidad de puntos de vista al momento de abordar una comprensión cabal de la realidad, siguiendo el hilo de las evidencias hasta donde éstas nos lleven.

Como hemos indicado, el Proyecto Delphi concluía identificando las habilidades intelectuales necesarias para el desarrollo del pensamiento crítico: Análisis, Inferencia, Interpretación, Explicación, Evaluación y Autorregulación. Por otro lado, los expertos llegaron al consenso de promover una disposición general a pensar críticamente basada en la idea de favorecer personas: inquisitivas; bien informadas; que confían en la razón; de mente abierta; flexibles; justas cuando se trata de evaluar; honestas cuando confrontan sus sesgos personales; prudentes al emitir juicios; dispuestas a reconsiderar y si es necesario a retractarse; claras con respecto a los problemas o las situaciones que requieren la emisión de un juicio; ordenadas cuando se enfrentan a situaciones complejas; diligentes en la búsqueda de información relevante; razonables en la selección de criterios; enfocadas en preguntar, indagar, investigar; persistentes en la búsqueda de resultados tan precisos como las circunstancias y el problema o la situación lo permitan. En suma, la disposición configura los aspectos de una curiosidad intelectual, una madurez cognitiva y una mente abierta.

Desde el denominado Proyecto Cero de la Universidad de Harvard, Tishman y colaboradores señalan que las disposiciones de pensamiento se aprenden a través 
de procesos culturales complejos más que por transmisión directa. Se entiende que al ser las disposiciones por naturaleza parte del carácter de la persona se desarrollan como muchos de los rasgos del carácter humano, teniendo en cuenta el ambiente cultural en que se encuentren. Esto supone una nueva vuelta de tuerca a la sistematización del pensamiento crítico y su medición.

En el marco específico de la práctica escolar existen diversas tentativas para canalizar la información y aplicación del pensamiento crítico. Por ejemplo, se han realizado estudios sobre el impacto del pensamiento crítico en el proceso de escolarización y sobre todo se han desarrollado programas para el desarrollo de la inteligencia, programas para estimular el razonamiento, tomar decisiones o solucionar problemas (los programas o guías para desarrollar el pensamiento crítico como FRISCO de Ennis [1996], el de Halpern [1998] o el de Lipman, "Filosofía para niños", el método CoRT de Bono, etc., son tan sólo algunos ejemplos representantivos).

En todos ellos se trata de favorecer habilidades y tareas de razonamiento, de resolución de problemas, de abstracción, de autoevaluación del pensamiento, etc., así como de carácter disposicional o actitudinal, asumiendo en todos la idea de que hay habilidades de pensamiento definibles e identificables, y que se pueden enseñar directamente ${ }^{14}$.

El principal problema o limitación que se plantea con el desarrollo de estos programas es que no siempre van seguidos por una integración curricular sustantiva, esto es, por lo común, son experiencias aisladas, propias de una materia o profesor determinado, sin la necesaria conexión con el resto de materias o profesores y sin un verdadero enfoque de enseñanza competencial ${ }^{15}$, lo que reduce en gran medida el impacto de los mismos.

Fuera del ámbito específico de la escuela, la aplicación del pensamiento crítico busca el desarrollo de ciudadanos autónomos y pensantes, responsables y solidarios, con un componente bastante más ideológico. En muchos casos se prefigura mediante la constitución de comunidades de aprendizaje en las que el diálogo se erige en el instrumento principal para el cuestionamiento propio y ajeno. Naturalmente, gran parte de este planteamiento tiene sus bases teóricas en la Teoría Crítica, desde la que se plantea un modelo educativo basado en procesos de transformación y no de adaptación.

Considerado en términos estrictamente pedagógicos, la formación de pensadores críticos se orienta aquí por el camino de los aspectos disposicionales y de lectura de la realidad. En el primer caso, interesa favorecer en el sujeto aspectos como la curiosidad epistemológica, la capacidad interrogativa, el entusiasmo por el descubrimiento. Desde el segundo, es fundamental el cuestionamiento permanente

14. Vid. SAIZ (2002).

15. Vid. Perrenoud (2009). 
LOS DOMINIOS DEL PENSAMIENTO CRÍTICO: UNA LECTURA DESDE LA TEORÍA DE LA EDUCACIÓN

del pensador crítico para analizar los procesos históricos y descubrir sus complejos entresijos.

En definitiva, los dominios del pensamiento crítico no sólo no delimitan el concepto sino que lo amplían hasta el punto de considerarlo desde el cuestionamiento sistemático de la lógica hasta del descubrimiento de los valores en la sociedad. En todo caso, parece claro que la expansión del concepto de pensamiento crítico se genera a partir de un acercamiento cognitivo y que su impacto en áreas afectivas y axiológicas nace de la amplia relación del conocimiento con la realidad.

\section{REFERENCIAS BIBLIOGRÁFICAS}

Althusser, L. (1975) Sobre la ideología y el estado. Ideología y aparatos ideológicos del Estado. Barcelona, Laia.

Bakunin, M. (2003) Dios y el Estado. Buenos Aires, Terramar Ediciones.

Benjamin, W. (1973) Tesis de la filosofía de la historia. Madrid, Taurus.

Сномsку, N. (1983) Teoría del aprendizaje. Barcelona, Grijalbo.

- (1988) Sobre el poder y la ideología. Madrid, Visor.

Costa, A. (1989) Toward the thinking curriculum: Current cognitive research, en ResNIK, L. B. y KLopfer, L. E. (eds.) ASCD yearbook. Alexandria, Association for Supervision and Curriculum Development.

Descartes, R. (2003) Discurso del método. Madrid, Tecnos.

Dewey, J. (1933) How we think. Boston, D. C. Health.

- (1938) Experience E Education. New York, Coier Book.

EnNIs, R. H. (1987) A taxonomy of critical thinking dispositions and abilities, en BARON, J. y SternBerg, R. (eds.) Teaching thinking skills: Theory and practice. New York, W. H. Freeman, 9-26.

- (1990) The extent to wich critical thinking is subject-specific: further clarification. Educational Researcher, 19, 13-16.

- (1993) Critical thinking assessment. Theory into practice, 32, 179-186.

Freire, P. (1997) A la sombra de este árbol. Barcelona, Editorial Roure.

Gabennesch, H. (2006) Critical Thinking: What Is It Good for? (In Fact, What Is It?), en http:// www.csicop.org/si/2006-02/thinking.html.

Gadamer, H. (1977 v. o. 1960) Verdad y método: fundamentos de una hermenéutica filosófica. Salamanca, Editorial Sígueme.

Giancarlo, C. A. y Facione, P. A. (2001) A look across four years at the disposition toward critical thinking among undergraduate students. The Journal of General Education, 50 (1), 29-55.

Giddens, A. (1985) The constitution of Society: Outline of the Theory of Structuration. Cambridge, Ing. Polity Press.

Glaser, E. (1941) An experiment in the development of critical thinking. New York, J. J. Little and Ives Company.

Grennan, W. (1997) Informal logic: issues and techniques. Montreal, McGill-Queen's University.

Habermas, J. (1984) Teoría de la acción comunicativa, I. Madrid, Editorial Taurus.

- (1989) El discurso filosófico de la modernidad. Madrid, Taurus.

- (1989) La modernidad: Un proyecto incompleto. Buenos Aires, Ediciones Punto Sur. 
- (1990) La Lógica de las Ciencias Sociales. Barcelona, Editorial Tecnos.

- (2000) La constelación postnacional. Barcelona, Paidós.

Halpern, D. F. (1998) Teaching critical thinking for transfer across domains. American Psychologist, 53 (4), 449-455.

Herrick, J. A. (1991) Critical thinking: the analysis of arguments. Scotts Dale, Arizona, Goursuch Scarisbrick.

Kant, I. (1993) ¿Qué es la Ilustración? (3. . ed.). Colección Clásicos del Pensamiento, vol. 43, Respuesta a la pregunta por la Ilustración. Madrid, Editorial Tecnos.

Kurland, C. (1995) Pensamiento Crítico. Barcelona, Eduketa.

Lipman, M. (1997) Pensamiento complejo y Educación. Madrid, Ediciones de la Torre.

Lipman, M. et al. (1992) La filosofía en el aula. Madrid, Ediciones de la Torre.

Marzano, R. J.; Brand, R.; Hughes, C. et al. (1988) Dimensions of thinking. VA, ASCD.

Montessori, M. (1921) Antropología Pedagógica. Barcelona, Araluce.

- (1965) Ideas generales sobre mi método. Buenos Aires, Losada.

Nietzsche, F. (1990) Sobre la verdad y mentira en sentido extramoral. Madrid, Tecnos.

PAUL, R. (1992) Teaching critical reasoning in the strong sense: getting venid worldviews, en TALASKA, A. R. Critical reasoning in contemporany culture. New York.

Perkins, D.; Jay, E. y Tishman, S. (1993) New Directions in the teaching of thinking. Educational Psychologist, 28 (1), 1-6.

Perrenoud, Ph. (2009) Enfoque por competencias, ¿una respuesta al fracaso escolar? Pedagogia Social, Revista Interuniversitaria, 16, 45-64.

Pestalozzi, J. H. (1946) Cartas completas. Zurich, Orell.

Proudhon, P. J. (1977) Solución del problema social. Madrid, Alfonso Durán.

Racofal, R. (1990) Logic for an overcast Tuesday. Belmont, Calif., Wadsworth.

Rogers, C. (1975) Libertad y creatividad en la educación. Buenos Aires, Paidós.

Rousseau, J. J. (1973) Emilio o de la educación. Barcelona, Fontanella (publicado originalmente en 1762).

Rudinow, J. y BARRY, V. E. (1994) Invitation to critical thinking (3. ${ }^{a}$ ed.). Harcourt Brace College, Inc.

SAIZ, C. (2002) Enseñar o aprender a pensar. Escritos de Psicología, 6, 53-72.

Schaff, A. (1973) Language and Cognition. Ed. Robert S. Cohen. Nueva Cork, MacGraw-Hill.

Scriven, M. y Fisher, A. (1997) Critical thinking. Its definition and assessment. Norwich (UK), Centre for research in Critical Thinking/Edgepress.

Van Dijк, T. у Kinstch, W. (1983) Estrategias de comprensión discursiva. Buenos Aires, Paidós Comunicaciones.

VAn Dijk, T. (1980a) La ciencia del texto. Barcelona, Paidós.

- (1980b) Texto y contexto. Madrid, Cátedra.

- (1983) Estructura y funciones del discurso. Una introducción interdisciplinar a la lingüistica del texto y a los estudios del discurso. Madrid, Siglo XXI.

Wittgenstein, L. (2000) Sobre la certeza. Barcelona, Gedisa.

- (2003) Tractatus lógico-philosophicus. Madrid, Tecnos. 